\title{
Circulating HMGB-1, HistoneH3, and Syndecan-1 in a Newborn with Neonatal Cerebral Infarction
}

\author{
Toshihiko Nakamura ${ }^{1 *}$, Tomoaki Nomura ${ }^{1}$, Shingo Yamada ${ }^{2}$, Daisuke Hatanaka ${ }^{1}$ Michiko \\ Kusakari $^{1}$ and Hidehiro Takahashi ${ }^{1}$ \\ ${ }^{1}$ Department of Neonatology, Japanese Red Cross Musashino Hospital, Musashino, Japan \\ ${ }^{2}$ Division of Development, Shino-Test Corporation, Kanagawa, Japan
}

*Corresponding author: Toshihiko Nakamura, Department of Neonatology, Japanese Red Cross Musashino Hospital, 1-26-1

Kyonan-cho, Musashino, Tokyo, Japan

\section{ARTICLE INFO}

Received: 慧 October 12, 2021

Published: October 20, 2021

Citation: Toshihiko Nakamura, Tomoaki Nomura, Shingo Yamada, Daisuke Hatanaka, Michiko Kusakari, et al., Circulating HMGB-1, HistoneH3, and Syndecan-1 in a Newborn with Neonatal Cerebral Infarction. Biomed J Sci \& Tech Res 39(3)-2021. BJSTR. MS.ID.006309.

\begin{abstract}
One of two late preterm twins at gestational age 36 weeks suffered idiopathic cerebral infarction. The first symptom was an apneic attack about 3 hours after birth. The lesion was extensive in the left middle cerebral artery region. Cord blood and early postnatal serum levels of high mobility group box-1 (HMGB-1), histoneH3, and syndecan-1 were measured simultaneously. All values of HMGB-1 remained within the reference value range. HMGB-1 and histoneH3 showed a very strong positive correlation $(\mathrm{r}=0.956)$. Syndecan-1 was detected but did not show a significant correlation with either HMGB-1 or histoneH3. This twin was delivered by Caesarean section before the first labor pains, and it was suspected that the ischemia-reperfusion injury that occurs at birth was minimal. Based on the serial changes of HMGB-1 in the early postnatal period, including in the umbilical cord blood, it is highly possible that the cerebral infarction had already occurred before birth. This case suggests that measurement of these three biomarkers released into the blood by ischemic reperfusion injuries such as stroke may be also useful for predicting these onsets.
\end{abstract}

Keywords: high mobility group box-1; histoneH3; ischemia-reperfusion injury; neonatal stroke; syndecan-1

\section{Introduction}

Neonatal cerebral infarction is a relatively rare central nervous system disorder that occurs in about 1 in 5000 neonates. The most common cause is ischemic cerebral injury resulting from neonatal asphyxia but can also be idiopathic. Half of the infarctions develop by the day 1 , and few occur over the 3rd day of life. Most occur in the middle cerebral artery region, most often on the left side [13]. Convulsions are the most common initial symptom, but there are many non-specific symptoms such as decreased feeding ability and apneic attacks. Treatment is mainly systemic management and symptomatic treatment for convulsions. Currently, there are only a few reported cases of the use of thrombomodulin, tissue plasminogen activator, and Edaravone (free radical scavenger), of which treatment method has been useful in adults [4], probably because of the many side effects for neonates. Neonatal cerebral infarction is currently classified into six categories from the viewpoint of pathogenesis, and most of the idiopathic cerebral infarctions belong to the category of ischemic cerebral infarction $[1,2]$. Similar cerebral ischemia and subsequent inflammation are pathological conditions of cerebral infarction in both newborns and adults, and the indication for treatment depends on how many hours have passed since the onset of cerebral infarction. In newborns, infarct lesions may appear shortly after birth or may have already occurred before birth and may be diagnosed by the detection of postnatal symptomsin the infant. Therefore, it is very difficult to determine the onset of neonatal cerebral infarction compared to that of adults. 
We have confirmed and reported in a multicenter cohort study that the effectiveness of brain hypothermia in neonatal asphyxia can be judged by changes in the serum high mobility group box1 (HMGB-1) concentration [5]. Furthermore, we observed the serial changes of HMGB-1 in the blood of infants who had already developed fetal asphyxia and suffered severe sequelae even though brain hypothermia was started within 6 hours after birth. We found that a long time had passed following the onset of ischemic lesions and reported that postnatal brain hypothermia may be ineffective for such hypoxic ischemic encephalopathy within utero onset [6]. This time we experienced a case of cerebral infarction in one of two twins. We had the opportunity to simultaneously measure three biomarkers, HMGB-1, histone H3, which is a nuclear protein similar to HMGB-1, and syndecan-1, which is present on the surface of vascular endothelial cells and is thought to be released in the blood during angiopathy. As a result, we report a case in which the onset of cerebral infarction was suspected to have occurred before birth.

\section{Case Presentation}
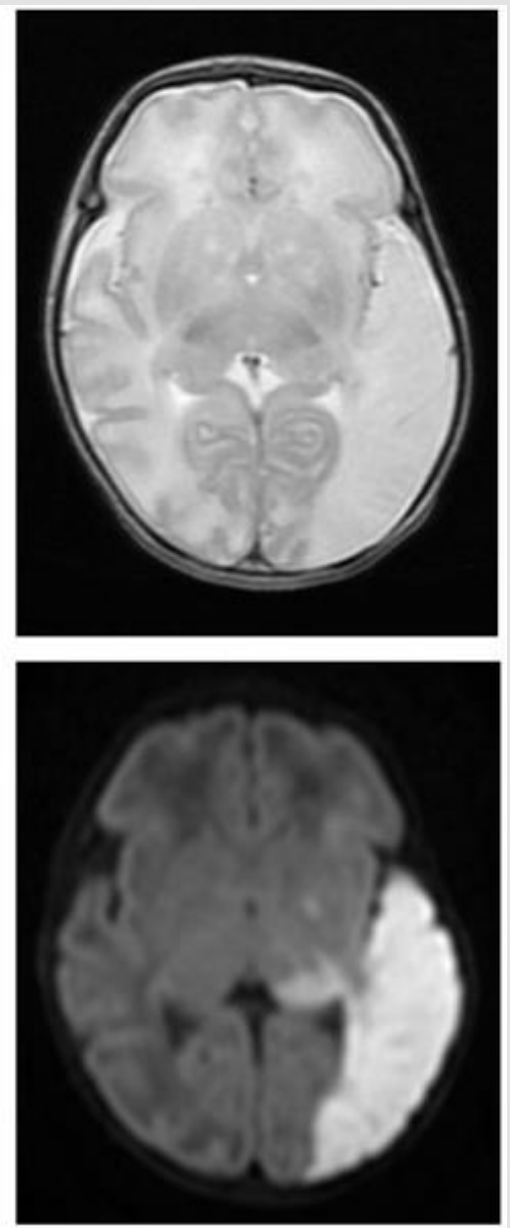

Figure 1: Magnetic resonance imaging at the $2^{\text {nd }}$ day of age above: T2-weighted image, below: Diffusion weighted image; T2-weighted images revealed extensive cerebral infarction in the left middle cerebral arteryregion.
The mother was 35 years old and had one pregnancy and zero deliveries. She had preeclampsia and was indicated for an emergency caesarean section due to exacerbation of her hypertension on the 36 th week, $3^{\text {rd }}$ day of her pregnancy. A female with a birth weight of $2420 \mathrm{~g}$ was born as the second baby of a diamniotic dichorionic twin pregnancy with an Apgar score of 8-9 and umbilical arterial $\mathrm{pH}$ of 7.273. At 2 hours and 54 minutes after her birth, she was admitted to the NICU due to an apneic attack. The infant was given intravenous phenobarbital for pedaling-like and muffled mouth movements on day 1 after birth, but it was ineffective and was changed to continuous administration of midazolam on day 2. Head echo showed no obvious lesions, but computed tomography and magnetic resonance imaging showed extensive cerebral infarction in the left middle cerebral artery region (Figure 1). The patient was diagnosed as having idiopathic cerebral infarction because a coagulation system test, amino acid fraction, and ophthalmologic examination were all negative. Midazolam was used from 2 to 8 days of age. No particular abnormalities such as in oral feeding and muscle tone were observed. An electroencephalogram was performed on day 22, and a decrease in activity on the left side was observed, but no obvious seizures were observed, so the patient was discharged from the hospital on day 31 after birth.

\section{Material and Methods}

\section{Measurement of HMGB-1 Levels}

The HMGB-1 measurements were performed by technicians with no knowledge of the personal data of the patient providing the samples using a commercially available ELISA kit (Shino-Test Corporation, Sagamihara, Japan). The detection sensitivity of this assay system was $0.2 \mathrm{ng} / \mathrm{mL}$ [7].

\section{Measurement of HistoneH3 Levels}

Because there is no commercially available ELISA kit, the measurement of histone H3 was performed by the ELISA prepared in our laboratory. The following is the method which had been presented in our laboratory was adopted this time as well [8]. Polystyrene microtiter plates (Nunc, Roskilde, Denmark) were coated with $100 \mu \mathrm{L} /$ well of $1 \mathrm{mg} / \mathrm{L}$ anti-histone $\mathrm{H} 3$ peptide polyclonal antibody (Shino-Test Corporation) in phosphatebuffered saline (PBS), and incubated overnight at $2-8^{\circ} \mathrm{C}$. After three washes with PBS containing 0.05\% Tween-20 (washing buffer), the remaining binding sites were blocked by incubation with 400 $\mu \mathrm{L} /$ well of PBS containing 1\% bovine serum albumin (BSA) for $2 \mathrm{~h}$. The plates were washed again and incubated with $100 \mu \mathrm{L} /$ well of diluted calibrator and serum samples (1:10 dilution in 0.2 $\mathrm{mol} / \mathrm{L}$ Tris $\mathrm{pH} 8.5,0.15 \mathrm{~mol} / \mathrm{L} \mathrm{NaCl}$, and $1 \% \mathrm{BSA}$ ) for $24 \mathrm{~h}$ at room temperature. After washing, the plates were incubated with $100 \mu \mathrm{L} /$ well of anti-histoneH3 peroxidase-conjugated peptide polyclonal antibody (Shino-Test Corporation) for 2 hours at room temperature. The plates were washed again, and the chromogenic substrate 
3,3',5,5'-tetra-methylbenzidine (TMBZ; Dojindo Laboratories, Kumamoto, Japan) was added to each well. The reaction was terminated with $0.35 \mathrm{~mol} / \mathrm{L} \mathrm{Na}_{2} \mathrm{SO}_{4}$, and the absorbance at 450 $\mathrm{nm}$ was measured with a microplate reader (Model 680; Bio-Rad, Hercules, CA, USA). A standard curve was obtained with purified calf thymus histoneH3 (Roche, Stockholm, Sweden). The amino acid sequence of histone $\mathrm{H} 3$ is highly conserved throughout species, and that of the antibody recognition in humans, calves, mice, and rats. This ELISA specifically detects histone $\mathrm{H} 3$ and does not react with other histone family proteins, including histone $\mathrm{H} 2 \mathrm{~A}, \mathrm{H} 2 \mathrm{~B}$, and $\mathrm{H} 4$, even if 104 times excess proteins are loaded. The detection sensitivity of this assay system was $2.0 \mathrm{ng} / \mathrm{mL}$.

\section{Measurement of Syndecan-1 Levels}

The following is the ELISA method which was presented originally in our laboratory. Polystyrene microtiter plates (Nunc, Roskilde, Denmark) were coated with $100 \mu \mathrm{L}$ anti-syndecan-1 monoclonal antibody (R\&D Systems) in PBS, and the plates were sealed with a thin adhesive-coated plastic sheet and incubated overnight at $37^{\circ} \mathrm{C}$. The unbound antibodies were removed by washing the plate 3 times with PBS containing $0.05 \%$ Tween 20 , and the remaining binding sites in the wells were blocked by incubating the plates for $2 \mathrm{~h}$ with $400 \mu \mathrm{L} /$ well of PBS containing $1 \%$ BSA. After washing, $100 \mu \mathrm{L}$ of each dilution of the standard and samples in $0.2 \mathrm{~mol} / \mathrm{L}$ Tris $\mathrm{pH} 7.4$ and $0.15 \mathrm{~mol} / \mathrm{L} \mathrm{NaCl}_{2}$ containing $1 \%$ BSA was added to the wells. The samples and recombinant syndecan-1 standard were diluted 1:10. The microtiter plates were incubated for 20-24 hours at room temperature. After washing, $100 \mu \mathrm{L}$ per well of anti-human syndecan-1 peroxidase-conjugated polyclonal antibody (R\&D Systems) was added, and the plates were incubated at room temperature for $2 \mathrm{~h}$. After washing, TMBZ (Dojindo Laboratories, Kumamoto, Japan) was added to each well. The enzyme reaction was allowed to proceed for $30 \mathrm{~min}$ at room temperature. The chromogenic substrate reaction was stopped by

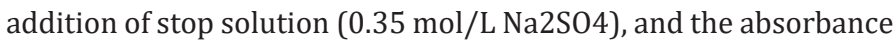
was read at $450 \mathrm{~nm}$.

\section{Ethical Approval}

This study was approved by the ethics committees of the Japanese Red Cross Musashino Hospital (\#28060). Parents of the twins were informed of the study design, and their written informed consent was obtained

\section{Results}

HMGB-1 and histone H3, which are common substances as nuclear proteins, showed a fairly strong positive correlation with a correlation coefficient of $r=0.965$ (Figure 2). Syndecan- 1 was low in both twins at each measurement, and no significant correlation was observed between HMGB-1 and histone H3 as previously reported [14]. HMGB-1 and histone H3 showed no significant variation in their levels in the specimens obtained before and after the onset of the first apneic attack (Figure 3).

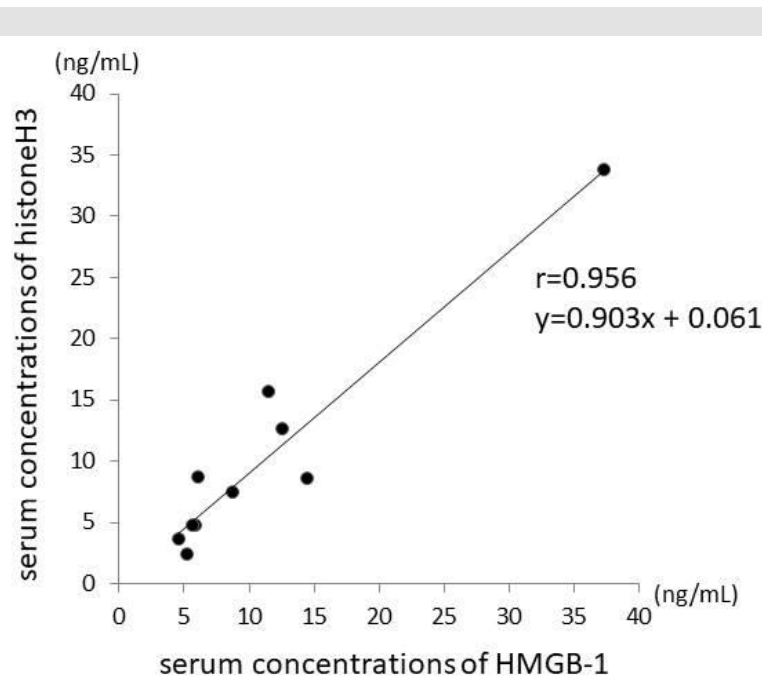

Figure 2: Correlations between serum HMGB-1 and histone $\mathrm{H} 3$ levels. A significant correlation was observed between HMGB-1 and histone H3 ( $\mathrm{r}=0.965, \mathrm{p}<0.0001)$. However, no significant correlations were found between syndecan-1 and HMGB-1 ( $p=0.431$ ) or histone H3 ( $p=$ $0.373)$.

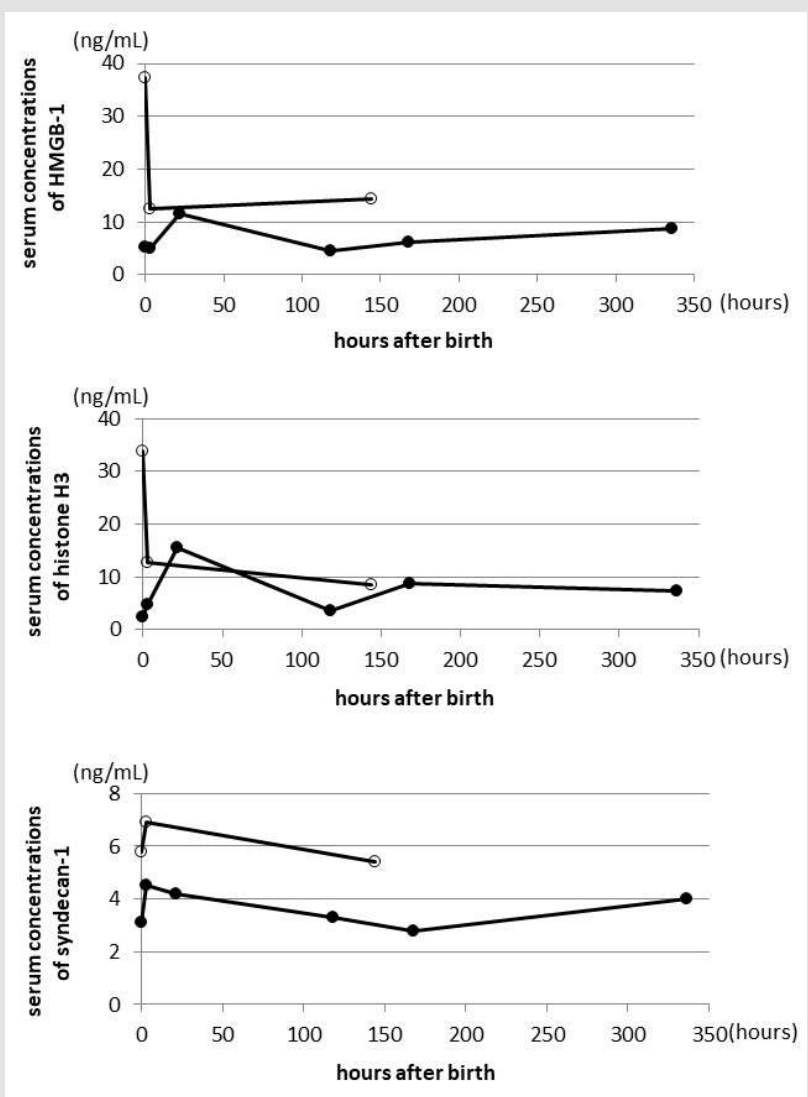

Figure 3: Serial changes of serum concentrations of HMGB1 , histone $\mathrm{H} 3$, and syndecan- 1 in this case twins.

: this case twin, $\bigcirc$ : this case co-twin. 


\section{Discussion}

Treatment for ischemic lesions of the brain is more effective the sooner it can be initiated after ischemia onset. Within 6 hours after the onset of ischemic lesions, HMGB-1 released from injured cells disrupts the blood-brain barrier (BBB) [9]. Then, within 24 hours after lesion onset, peroxiredoxin released from the cells acts on macrophages migrating from the injured part of the BBB to release inflammatory cytokines such as IL-1 $\beta$ and TNF $\alpha$. Then, 24 hours after the onset, IL-17 and IFNץ are released from T cells, exacerbating damage to the BBB and brain cells $[4,10]$. The indication for brain hypothermia to treat neonatal hypoxic-ischemic encephalopathy is "the treatment can be started within 6 hours after birth", and that for Edaravone ${ }^{\circ}$ a free radical scavenger, as a treatment for cerebral infarction in adults is "the treatment should start within 72 hours of onset". These indications make sense in terms of protecting against this inflammatory response after temporal ischemia. In adults, the origin of onset is often clear, but in newborns, onset does not always occur at birth. In our previous case, severe ischemic encephalopathy had already occurred before birth, and the ischemic encephalopathy became severe even though brain hypothermia was performed within 6 hours after birth [6]. The serum cytokine profile and HMGB-1 level in this case was measured over time to develop a theoretical diagnosis and report. We consider that asphyxia is a condition of systemic ischemiareperfusion and cerebral infarction is a condition of local ischemiareperfusion.

Both HMGB-1 and histoneH3 are nuclear proteins. It was expected that the blood concentrations of these two substances would correlate, as they would be released into the blood when the cells were injured. Although the number of samples obtained was small, the values showed a very strong positive correlation $(r=$ 0.965). In contrast, the serum HMGB-1 levels in this twin remained within reference values we have already reported for all cord blood and early postnatal specimens [7]. HistoneH3, which showed a strong correlation, was also about the same value. As this baby was born by a caesarean section before labor, it was inferred that the so-called "physiological ischemia-reperfusion stress" associated with birth would be minimal [11]. In addition, the changes in the HMGB-1 level suggested that the probability of developing cerebral infarction from immediately after birth to the onset of the apnea was extremely low. Considering that the apneic attack occurred early after birth, it is highly possible that the onset of cerebral infarction in this baby occurred prenatally and that most of the injury process was already completed by the time of birth. This suggested a reason why the HMGB-1 and histoneH3 levels in the cord blood at birth and as measured after birth did not increase $[12,13]$.

Syndecan-1 is a representative proteoglycan expressed on the surface of vascular endothelial cells. So far, it has been reported that syndecan-1 is released into the bloodstream due to vascular endothelial cell damage in the early stage of sepsis [14]. Furthermore, it has been confirmed to increase in an ischemic perfusion injury model used in the animal experiment of Gayosso et al. [15]. Syndecan-1 did not significantly correlate with HMGB-1 and histone H3. This result was consistent with the report in adults by Ikeda et al. To the best of our knowledge, the Ikeda et al. [14] report, which focused on sepsis rather than cerebral infarction, is the only report of the simultaneous measurement of the three biomarkers HMGB-1, histone H3, and syndecan-1. The presentcase may be the second report and especially the first report in newborns. When released extracellularly, HMGB-1 not only directly damages the BBB but also acts on monocytes to increase the expression level of tissue factor and promote fibrin production by thrombin, thereby promoting thrombus formation [4]. Histone H3, a structure of NETs (neutrophil extracellular traps) released from neutrophils, is also involved in thrombus formation when released extracellularly [8]. Furthermore, syndecan- 1 is also exfoliated and released into the bloodstream when vascular endothelial cells are damaged, and a thrombus is formed on the surface of the vascular endothelial cells [14]. If it can be confirmed that the levels of these three substances, which are released in common into the blood stream due to cells damaged by ischemia-reperfusion injury, are elevated in the acute phase, the use of drugs such as thrombomodulin and Edaravone ${ }^{\circledR}$ may be effective for neonatal cerebral infarction. Future accumulation of additional cases and simultaneous measurement of these three biomarkers must be useful for determining the onset of cerebral infarction.

\section{Conflict of Interest Statement}

The authors have no conflicts of interest to declare.

\section{References}

1. Dunbar M, Kirton A (2019) Perinatal Stroke. Semin. Pediatr. Neurol 32: 1-11.

2. Rutherford MA, Ramenghi LA, Cowan FM (2012) Neonatal Stroke. Arch Dis Child Fetal Neonatal Ed 97(5): 377-F384.

3. Talib TL, Pongonis SJ, Williams LS, Garg BP, Sokol DK, et al. (2008) Neuropsychologic outcomes in a case series of twins discordant for perinatal stroke. J Pediat Neurol 38: 118-125.

4. Shichita T, Ito M, Yoshimura A (2014) Post-ischemic inflammation regulates neural damage and protection. Front Cell Neurosci 8. 319.

5. Nakamura T, Asanuma H, Kusuda S, Imai K, Hosono S, et al. (2017) Multicenter study for brain/body hypothermia for hypoxic-ischemic encephalopathy: Changes in HMGB. Pediatr Int 59(10): 1074-1079.

6. Nakamura T, Nomura T, Takizawa Y (2018) Significantly increased levels of serum high mobility group box 1 and interleukin 17 in a female neonate with antepartum severe hypoxic ischemic encephalopathy due to placental abruption. J Jpn Soc Neonat Heal Dev 30: 33-41.

7. Nakamura T, Yamada S, Yoshioka T (2011) Measurement of plasma concentration of high mobility group box-1 (HMGB1) in early neonates and evaluation of its usefulness. Clin. Chim. Acta 413(1-2): 237-239. 
8. Ito T, Nakahara M, Masuda Y, Ono S, Yamada S, et al. (2018) Circulating histone $\mathrm{H} 3$ levels are increased in sepitic mice in a neutrophil-dependent manner: preclinical evaluation of a novel sandwich ELISA for histone H3. J Intensive Care 6: 79-85.

9. Qiu J, Nishimura M, Wang Y, Sims JR, Qui S, et al. (2008) Early release of HMGB-1 from neurons after the onset of brain ischemia. J Cereb Blood Flow Metab 28(5): 927-938.

10. Shichita T, Hasegawa E, Kimura A, Morita R, Sakaguchi R, et al. (2012) Peroxiredoxin family proteins are key initiators of post-ischemic inflammation in the brain. Nat Med 18: 911-917.

11. D Angelo G, Marseglia L, Granese R, Di Benedetto A, Giacobbe A, et al. (2018) Different concentration of human cord blood HMGB-1 according to delivery and labor: A pilot study. Cytokine 108: 53-56.

ISSN: 2574-1241

DOI: 10.26717/BJSTR.2021.39.006309

Toshihiko Nakamura. Biomed J Sci \& Tech Res

(C) (P) This work is licensed under Creative

Submission Link: https://biomedres.us/submit-manuscript.php
12. Bell JD, Rhind SG, Di Battista AP, Macdonald RL, Baker AJ, et al. (2017) Biomarkers of glycocalyx injury are associated with delayed cerebral ischemia following aneurysmal subarachnoid hemorrhage: A case series supporting a new hypothesis. Neurocrit Care 26(3): 339-341.

13. Chen X, Zhang J, Kim B, Jaitpal S, Meng SS, et al. (2019) High-mobility group box-1 translocation and release after hypoxic ischemic brain injury in neonatal rats. Exp Neurol 311: 1-14.

14. Ikeda M, Matsumoto H, Ogura H, Hirose T, Shimizu K, et al. (2018) Circulating syndecan-1 predicts the development of disseminated intravascular coagulation in patients with sepsis. J Crit Care 43: 48-53.

15. Rubio-Gayosso I, Platts SH, Duling BR (2006) Reactive oxygen species mediate modification of glycocalyx during ischemia-reperfusion injury. Am J Physiol Heart Circ Physiol 290(6): H2247-H2256.

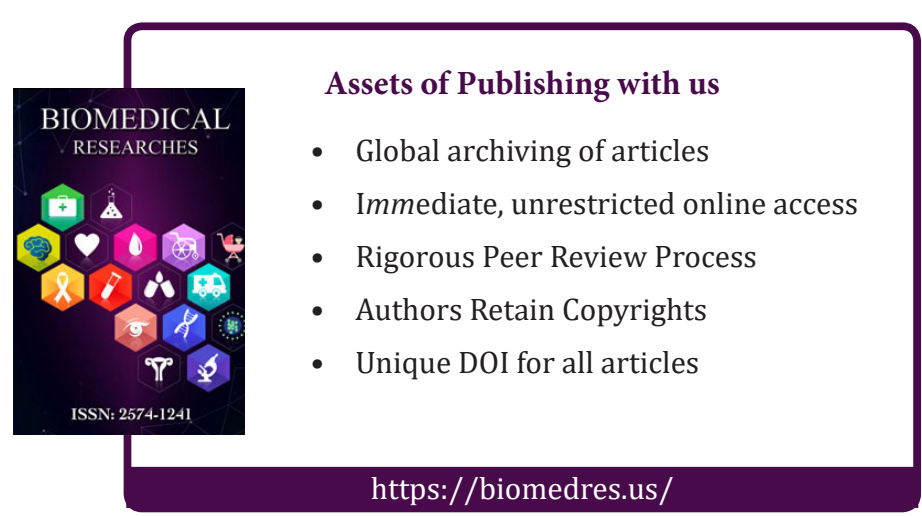

